\title{
La educación secundaria como objeto de investigación y de políticas públicas. Actualizaciones y debates
}

\section{Presentación de la segunda parte del dossier "Estudios y debates sobre educación secundaria"}

\author{
( Flavia Terigi \\ Universidad de Buenos Aires / Universidad Nacional de General Sarmiento, Argentina \\ fterigi@campus.ungs.edu.ar
}

\begin{abstract}
Damos continuidad en este número de la Revista al dossier dedicado a la investigación sobre Educación Secundaria. En esta segunda parte, el dossier reúne trece trabajos que actualizan el estado de situación en la universalización del nivel, documentan algunas políticas educativas que se proponen transformarlo a partir de procesos institucionales, y presentan investigaciones que profundizan en los rasgos que toma la escolarización secundaria en la actualidad, entre ellos las decisiones de enseñanza y las características de la experiencia de formación que estaría produciéndose en las instituciones de este nivel escolar.
\end{abstract}

Todos los trabajos que componen esta segunda entrega han sido producidos por investigadorxs de Argentina, tanto de universidades estatales como de dos unidades de investigación dependientes de órganos de gobierno. Seis de los trece trabajos surgen de tesis doctorales, dos de ellas en curso, lo que ilustra el interés de la escuela secundaria como objeto de investigación y los incentivos que han existido en los últimos años para que se convirtiera en asunto de proyectos de becas académicas entre lxs investigadores en formación.

Dos temáticas que ya fueron tratadas en la primera parte son aquí objeto de nuevas producciones: la enseñanza en la educación secundaria, y la secundaria como experiencia de formación. Una mirada de conjunto de ambas requiere considerar los cinco artículos sobre la primera y otros tantos sobre la segunda, distribuidos entre las dos partes que componen el dossier. Junto con ello, en esta oportunidad, incorporamos tres temáticas que no fueron tratadas de manera específica en el número 46:

» Sistematización y segmentación: dinámica histórica y expresiones actuales

»El ciclo de las políticas: miradas sobre los procesos de las instituciones escolares en Argentina

» Saberes clásicos y nuevos saberes

A continuación, presentamos los cinco organizadores temáticos de esta segunda parte del dossier, junto con los trabajos que los integran. 


\section{Sistematización y segmentación: dinámica histórica y expresiones actuales}

Integran este apartado dos trabajos que ubican las dinámicas de la educación secundaria en la escala del sistema escolar y que, aunque situados en la educación secundaria en Argentina, tienen la virtud de referir sus asuntos a la escala global. La investigación situada en establecimientos específicos, o centrada en políticas determinadas, o que se inicia a partir de problemas formulados en otros campos, deja la preocupación, a quienes investigamos sobre educación secundaria, acerca de en qué medida los estudios -que son "sobre Secundaria" porque realizan su trabajo de campo en establecimientos del nivel, o con docentes que trabajan en el nivel, o con adolescentes y jóvenes que son/ no son estudiantes del nivel- incorporan a su modo de definir y estudiar sus asuntos los aportes de las investigaciones especializadas sobre este objeto. Además del claro diálogo que establecen con estos análisis, un interés que seguimos al agrupar estos dos trabajos es que permiten poner en discusión el alcance y las limitaciones de las políticas de expansión del sistema educativo que, como venimos advirtiendo desde hace tiempo (Terigi, 2008), suelen definirse sin analizar los modelos institucionales sobre los que se propone la expansión.

El primer trabajo, "La sistematización estatal modelizadora y la segmentación en los orígenes y expansión de la escuela secundaria en la Argentina", de Felicitas Acosta, debería convertirse en una referencia ineludible en las futuras investigaciones sobre este nivel escolar. Es un artículo que contribuye a la discusión sobre cuestiones que son parte del debate académico actual, que utiliza categorías teóricas relevantes y que tiene implicancias para comprender algunos problemas del diseño de políticas públicas. Acosta analiza el proceso de sistematización de la educación secundaria en el país con herramientas de trabajo (conceptos como sistematización, segmentación, diferenciación, institución determinante) que provienen de los estudios histórico-comparativos sobre el desarrollo de los sistemas educativos y las instituciones de educación secundaria. El trabajo parte de la hipótesis de que la expansión de la escuela secundaria argentina se produjo sobre la base de una dinámica de incorporación y expulsión simultánea, en la que el modelo institucional de origen (el colegio nacional como "institución determinante") parece haber ocupado un lugar central y haber tenido efectos en la forma que adoptó la segmentación durante la expansión de la escuela secundaria. Según propone Acosta, el Estado nacional, a través de la creación y modificación de distintos tipos de oferta destinada a la "segunda enseñanza", llevó a cabo un proceso de sistematización sobre la base de una tendencia modelizadora, que la autora propone que estaría asociada a las formas que adoptó la segmentación en el sistema educativo argentino: segmentación por diferenciación institucional. "Hubo un modelo institucional determinante", nos dice Acosta, "pero su formación y perfeccionamiento durante la expansión fue producto de hibridaciones que buscaron al mismo tiempo proveer una oferta generalista y resguardar espacios de jerarquía cultural".

El artículo no es un ensayo: utiliza fuentes primarias (como decretos y reglamentos oficiales) y fuentes secundarias y -nos consta- se apoya en un riguroso esfuerzo por construir series estadísticas de largo plazo, para lo cual la tesis doctoral que lo sustenta debió resolver los problemas que genera que las categorías para definir el objeto y los modos de recolección de la información no sean sistemáticos a lo largo del extenso proceso histórico que se considera. Cuando lxs lectores analicen la estadística que propone Acosta, recuerden que no tiene sentido evaluar la evolución de la cobertura de la educación secundaria desde el imperativo contemporáneo de la universalización (apoyado normativamente por la obligatoriedad), pues se trata de un largo periodo en el que la expansión parece producto de la combinación de la presión de la población por prolongar la escolaridad de sus hijxs con la iniciativa estatal que abre establecimientos de nivel secundario y expande la planta docente (Terigi, 2020, en prensa). 
En el proceso histórico que analiza Acosta, en la educación secundaria argentina se verifica la expansión primero (a lo largo de varias décadas) y la obligatoriedad después (con dos ampliaciones de la obligatoriedad: primero, la de la EGB, luego, la del completamiento de la Secundaria). Hace más de un cuarto de siglo que se prolongó la obligatoriedad escolar por primera vez (Ley 24195) y hace catorce que se determinó la finalización de la escuela secundaria como el término de la educación obligatoria (Ley 26206). Un análisis que nos parece necesario es el que se interroga acerca de la factibilidad del tránsito de una escuela de élite a una escuela de masas o, expresado de otro modo, acerca de la capacidad del modelo prevaleciente de educación secundaria de hacer efectiva la incorporación de capas medias y sectores populares. Ese problema encuentra un análisis posible en el trabajo de Bárbara Briscioli y Victoria Rio, "La transición de primaria a secundaria en la provincia de Buenos Aires en el escenario de la extensión de la obligatoriedad escolar. Inclusión y diferenciación educativa". Las autoras colocan la transición entre el nivel primario y el nivel secundario - un proceso que suele ser interpretado como un problema entre escuelas y que se resuelve a nivel local- como un asunto de escala sistémica y mundial. Como señalan en su trabajo, la acelerada extensión de los años de escolaridad y el creciente mayor acceso de la población a los niveles medio y superior que tuvieron lugar en la segunda mitad del siglo $\mathrm{XX}$ a nivel mundial se dieron, entre otros elementos, mediante la revisión y dilución de algunas de las formas concretas de la diferenciación de trayectos escolares, tanto en su dimensión horizontal, como en las políticas existentes de carácter selectivo o restrictivo en la transición entre niveles.

En el caso argentino, nos dicen las autoras, desde la década del ochenta (y, agregaremos nosotrxs, con la reinstauración de gobiernos constitucionales) comenzó a modificarse la política de transición al secundario, a través de un proceso de disolución de mecanismos restrictivos de acceso al nivel. En la provincia de Buenos Aires, territorio en el que se sitúa el trabajo de campo del texto de Briscioli y Rio, se estableció el derecho al ingreso sin formas selectivas. En el trabajo se analiza la forma que asume en la actualidad la transición de la primaria a la secundaria en seis instituciones de nivel secundario del conurbano bonaerense, para conocer algunas formas concretas en que se realiza. Para ello, se analizan tres elementos: las formas de ingreso a las escuelas secundarias del estudio, los proyectos de articulación que estas realizan y promueven, y algunos criterios pedagógicos que se ponen en juego para el primer año de la Secundaria.

El trabajo nos sugiere los modos en que se entretejen viejas, reconfiguradas y nuevas formas de diferenciación educativa en la transición/articulación entre los niveles primario y secundario del sistema educativo bonaerense. Las autoras plantean, "a modo exploratorio", una hipótesis (que puede analizarse en conjunto con la que plantea Acosta) según la cual ciertas prácticas que documentan constituyen formas de "inclusión diferenciada" y, si así fuera, dejan expresado que los mecanismos de diferenciación educativa tendieron a posponerse y/o a expresarse bajo nuevas formas, lo que -interpretamos nosotrxs- no puede explicarse únicamente por las lógicas provenientes de las matrices de origen específicas de cada uno de los niveles en cuestión.

\section{El ciclo de las políticas: miradas sobre los procesos de las institu- ciones escolares en Argentina}

En la introducción de la primera parte del dossier propusimos, recuperando una producción reciente (Terigi, 2016), considerar los tres componentes de la matriz organizacional de la escuela media/secundaria (un curriculum fuertemente clasificado, unos procesos de reclutamiento y formación de profesores según el principio de la especialidad disciplinar, y puestos de trabajo conformados como colecciones de tiempos 
rentados que espejan las horas de clase de los estudiantes) y el régimen académico como condiciones de escolarización que han funcionado históricamente como límites para la expansión y que imponen dificultades específicas en los tránsitos de adolescentes y jóvenes por el nivel. Las dos ampliaciones de la obligatoriedad de Secundaria en Argentina se definieron normativamente sin que se alteraran a escala del sistema aquellas condiciones. En investigaciones propias, hemos analizado el carácter de las innovaciones que propusieron las políticas educativas para el nivel secundario sobre las condiciones de escolarización (Terigi, Briscioli, Scavino, Morrone y Toscano, 2014), lo que nos permitió identificar dos tipos de iniciativas:

1. Iniciativas que propusieron innovaciones de carácter intensivo respecto de las condiciones de escolarización (por la cantidad y articulación de innovaciones propuestas respecto de la matriz organizacional del nivel y el régimen académico).

2. Iniciativas que proponen innovaciones de carácter extensivo (porque los cambios, aunque de gran escala, son reducidos en relación con la matriz organizacional existente).

Según esta distinción, hemos afirmado que las iniciativas universales, impulsadas y financiadas por el Estado nacional, no plantearon transformaciones en las condiciones de escolarización secundaria, aunque algunas iniciativas de menor escala sí lo han hecho. En cambio, algunas provincias avanzaron hacia propuestas intensivas en términos de las distintas dimensiones sobre las que intervinieron. Iniciativas jurisdiccionales más acotadas en escala (como las Escuelas de Reingreso, de la Ciudad Autónoma de Buenos Aires; ${ }^{1}$ el Programa de Inclusión y Terminalidad de la Escuela Secundaria y Formación Laboral para Jóvenes entre 14 y 17 años, de Córdoba; ${ }^{2}$ el plan Vuelvo a Estudiar, de Santa $\mathrm{Fe}^{3}$ ) alteraron de distintos modos la matriz organizacional y el régimen académico de la escuela secundaria. Son características de todas estas experiencias la reorganización interna de las instituciones, la flexibilización de tiempos y espacios, la creación de nuevas funciones en el ámbito institucional. El seguimiento de estas iniciativas es de interés para la investigación, porque puede contribuir a que comprendamos mejor cómo operan aquellas condiciones de escolarización y porque permite evaluar de qué manera los cambios que se introducen en ellas inciden en la experiencia escolar de los y las adolescentes y, eventualmente y como consecuencia, en el incremento de la cobertura, la retención y el egreso del nivel.

Los trabajos que agrupamos en este apartado comparten el interés por estudiar alguna iniciativa específica de política educativa, analizándola en la escala de algunas de las instituciones escolares en las que procuraron implantarse y en momentos específicos del desarrollo de la iniciativa. Como ya es conocido en los resultados de investigaciones sobre el desarrollo de las políticas educativas, estos estudios documentan las tensiones y contradicciones que conlleva la materialización de una política pública en un contexto específico, y acaban encontrando diferencias entre los enunciados de la política y sus concreciones en el terreno de los casos bajo estudio. Aunque no todos la adoptan, los aportes de los trabajos que agrupamos en este apartado pueden ser leídos desde la perspectiva del análisis de trayectorias o ciclos de vida de las políticas (Policy Cicle Approach) propuesto por Stephen Ball (véase Avelar, 2016), que procura superar las visiones lineales de las políticas como una producción acabada del Estado que las escuelas "implementan", y que enfatiza la complejidad de influencias en juego

\footnotetext{
1 Decreto CABA 408/2004. Han sido objeto de distintas investigaciones (véase, por ejemplo, Arroyo y Poliak, 2011; Di Pietro y Abal Medina, 2013; Maddonni, 2014; Krichesky, Greco y Saguier, 2015).

2 Creado en 2010 y vigente al cierre de este trabajo, también ha sido estudiado en distintas investigaciones (véase Vanella y Maldonado, eds., 2013; Yapur, 2018).

3 En este plan -que se inició en 2013 y se encontraba vigente al cierre de este trabajo-, el gobierno de Santa Fe decidió “ir a buscar" a quienes habían dejado de estudiar para que retomasen y terminasen la Educación Secundaria obligatoria.
} 
en los muchos momentos en que se definen y redefinen las políticas educativas (Ball y Bowe, 1992). Entender la política como texto, discurso y como "puesta en práctica" ha contribuido a comprender las formas "creativas" en que docentes y directivos de escuela producen y son producidos por las políticas educativas en contextos socioinstitucionales y profesionales específicos (Beech y Meo, 2016).

Los cambios en el régimen académico de la escuela secundaria forman parte de los textos políticos producidos desde 2009 en adelante. María Cecilia Bocchio y Silvia Grinberg estudian el Nuevo Régimen Académico de la Provincia de Córdoba ${ }^{4}$ en el trabajo "La trayectoria escolar asistida: entre el aula heterogénea, las pruebas Aprender y un poco de auto-ayuda. Tensiones cotidianas del Nuevo Régimen Académico para la escolaridad secundaria (Córdoba, Argentina)". Interesa el Nuevo Régimen Académico, lanzado de "modo piloto" por el Ministerio de Educación de la Provincia de Córdoba en 2018, como una expresión de las ideas que se debaten en el campo de la educación secundaria sobre las reglas que rigen la posición de los estudiantes. Bocchio y Grinberg señalan que los instrumentos de regulación institucional de la escolaridad secundaria están siendo reconfigurados de cara a flexibilizar el régimen académico y a generar condiciones para garantizar la obligatoriedad del nivel de enseñanza. En ese marco, estudian el proceso del Nuevo Régimen Académico en una de las 76 escuelas cordobesas que iniciaron en 2018 la puesta en acto de esta política; en el escrito se expone especialmente un encuentro de taller entre docentes y directivos de la escuela. Señalan, con apoyo en el material de campo de su estudio, que "la trayectoria escolar asistida", como mecanismo de inclusión, es tensionada por diversos instrumentos de evaluación, entre ellos, la evaluación estandarizada y externa, como las pruebas Aprender. ${ }^{5}$ Ante tales tensiones, documentan la búsqueda de alternativas docentes; aparecen en la escena escolar en el caso bajo estudio lo que denominan "relatos de auto-ayuda", como modos de afrontar el desafío de garantizar la inclusión apelando a las voluntades individuales de lxs docentes sobre lo que se quiere y lo que se puede hacer por los alumnos.

Armando Belmes e Ismael Álvaro Rodrigo presentan, en su artículo “Terminalidad de la educación secundaria y formación para el trabajo en la Ciudad de Buenos Aires: el Bachillerato con Orientación Profesional", algunos de los resultados de un estudio exploratorio que examina un dispositivo de inclusión educativa, el Bachillerato con Orientación Profesional, destinado a adolescentes que, por distintas circunstancias asociadas a la vulnerabilidad social y educativa, han transitado trayectorias discontinuas en el sistema educativo. Este dispositivo se implementa desde 2015 en seis Centros de Formación Profesional de la Ciudad de Buenos Aires y, en el texto de la política, se afirma la importancia de articular la enseñanza de las asignaturas básicas de la formación general de nivel Medio con un trayecto curricular de Formación Profesional inicial correspondiente a un sector de actividad específico.

El estudio analiza la estructura curricular y la organización institucional del dispositivo, y explora las particularidades de su implementación en cada uno de los centros. Los autores señalan, a la par del trabajo "artesanal" docente y el profundo compromiso de los actores institucionales, una brecha entre el enunciado y la práctica, cierta "distancia entre la formulación del dispositivo y su implementación", que se revela en dos aspectos: la tensión flexibilidad vs grupalidad, generada por un régimen académico flexible que tiene como contracara la desarticulación del formato contenedor del grupo clase homogéneo, "que hace pie en la experiencia acumulada en la educación de jóvenes y adultos"; y las dificultades para tender puentes entre la formación general y la formación

4 Córdoba. Ministerio de Educación de la Provincia de Córdoba. Resolución Ministerial 118/2018. Programa Nuevo Régimen Académico para la Escuela Secundaria y Régimen Académico de la Educación Secundaria de la Provincia de Córdoba. 
profesional, que evidencian las limitaciones para superar la fragmentación disciplinar tradicional de la escuela secundaria en general y en particular de la técnica-profesional.

En "El formato organizacional del Plan FinEs en la provincia de La Pampa: entre lo nuevo y lo viejo", Mariana Correa presenta algunos análisis realizados en el marco de un estudio de corte cualitativo de la primera etapa del Plan FinEs ${ }^{6}$ (2008-2011) en instituciones de General Pico y Eduardo Castex, en la provincia de La Pampa. En el trabajo se analizan las percepciones de los estudiantes egresados acerca de tres elementos que constituyen el "formato organizacional" del Plan FinEs: la organización del tiempo, la del espacio y la administración de los recursos. Volvemos a encontrar aquí el problema de la distancia entre la formulación de la política y su concreción en las instituciones: el trabajo de Correa hace evidente la persistencia de viejos formatos en propuestas que se presentan como alternativas/flexibles. A modo de ilustración de lo que puede consultarse con detalle en el trabajo, la autora señala que, si bien en los documentos ministeriales el FinEs se presenta como una "agenda didáctica flexible", adaptada a las necesidades de jóvenes y adultos (en la que el docente tutor y el estudiante acuerdan un plan y cronograma de producción de actividades de enseñanza y aprendizaje a lograr en ocho encuentros tutoriales y una evaluación final), su materialización en las sedes replica rasgos propios del formato tradicional de la escuela secundaria, con pocas variantes. Los/as egresados/ as valoran de manera positiva la ruptura de la clásica relación espacial asimétrica entre docente y estudiantes, pero también refieren que, en las sedes analizadas, los recursos disponibles son solo los proporcionados por las escuelas en las que se desarrolla el FinEs, y no por el programa, lo que contribuye a replicar experiencias de aprendizaje similares a las vivenciadas por los estudiantes en su paso fallido por la escuela secundaria común.

El trabajo de Gabriela Nacach, "Hacia una genealogía de la experiencia: La gestión escolar en una escuela de creación (2009-2015)", se recorta entre los otros que integramos en este apartado porque adopta un estilo personal y autorreflexivo, en sus propios términos "un estilo de escritura decididamente más narrativo -que se podría ajustar más a una crónica- que académico". Su trabajo surge de una praxis educativa entre 2009 y 2015 en una escuela secundaria "de creación" de la Ciudad Autónoma de Buenos Aires, una "Escuela de Expresiones Culturales" definida por la autora como un espacio de formación de un tipo de perfil de artista y comunicador "más ligado al establishment que a una visión del arte y los medios como herramientas para la transformación social". Sin embargo, el equipo de conducción que se hizo cargo de la creación de la escuela decidió abandonar el diseño propuesto por las autoridades y definir un Proyecto Educativo Institucional que contemplaba parejas pedagógicas, aulas por áreas, rol de coordinaciones como mediadores reales de la propuesta educativa, proyectos institucionales (las efemérides y los actos escolares como espacios de construcción conjunta del conocimiento sobre la historia), la elaboración con la comunidad escolar del código de convivencia, un fuerte vínculo con las familias y la cooperadora.

El trabajo expresa un valioso esfuerzo por considerar la propia experiencia en diálogo con conceptos teóricos del campo de la educación y de las ciencias sociales en general, aun con las comprensibles dificultades que ello supone, porque no se trata del modo predominante de producción de conocimiento, lo que entraña considerables dificultades para el análisis conceptual. Constituye un ejemplar interesante de algo que la etnografía educativa nos advierte desde hace décadas: que centrarse en un espacio micropolítico - "una institución, unas aulas, unos y unas jóvenes", nos dice la autorapone sobre la mesa la imposibilidad de repensar lo que acontece localmente fuera de un contexto sociopolítico, laboral y profesional más amplio. 


\section{La enseñanza en la educación secundaria}

En la primera parte del dossier incluimos tres artículos sobre la enseñanza en el nivel secundario; en esta segunda parte, incorporamos otros dos, que amplían el panorama que ofrece la Revista sobre la importancia de los estudios acerca de la enseñanza en el campo de investigaciones sobre educación secundaria.

En "La regulación metacognitiva sobre los modos de pensar en el aula de biología", Gastón Pérez y Leonardo González Galli nos introducen en la enseñanza de una asignatura clásica de la educación secundaria: la Biología. En su estudio, han trabajado con una secuencia didáctica sobre dos modelos de la biología evolutiva y, en el trabajo que nos presentan, se proponen caracterizar los procesos de regulación metacognitiva que emergieron durante una propuesta didáctica basada en la modelización y la metacognición sobre los obstáculos epistemológicos. Ese propósito se relaciona con una hipótesis de los autores que identifica, entre los problemas que entrañan la enseñanza y el aprendizaje de las ciencias, los modos de razonar de los sujetos (entre otros, el esencialismo o el determinismo), que se convierten en obstáculos epistemológicos. La originalidad del escrito reside en proponer una clasificación de los procesos por los que lxs estudiantes afrontan de los obstáculos epistemológicos, y en analizar los modos en que eventualmente lxs alumnxs los superan durante las secuencias didácticas. Los autores identifican tres modos de regular, que denominan el señalamiento del obstáculo epistemológico, la regulación individual compleja, y la regulación social compleja. Según afirman los autores, esta caracterización de las regulaciones puede ayudar a pensar en actividades útiles para mejorar el desarrollo de las habilidades metacognitivas.

La enseñanza se coloca también en el centro del trabajo de Egle Pitton y Fabiana Demarco, "Inclusión de estudiantes ciegos en el nivel secundario común de gestión estatal de la Ciudad de Buenos Aires". En tanto el estudio tiene como propósito comprender en qué sentidos las normativas y políticas que se sustentan en el actual paradigma de inclusión escolar -que promueve el derecho a la escolarización de todos los niños, niñas y adolescentes en la escuela "común"- inciden en las prácticas cotidianas de las instituciones educativas, de sus docentes y en la vida de niños y adolescentes con discapacidad que hoy asisten a instituciones estatales comunes de la Ciudad de Buenos Aires, podría ser analizado también con la ya citada perspectiva del análisis de trayectorias o ciclos de las políticas. Sin embargo, lo incluimos junto con los otros trabajos sobre la enseñanza porque, en el campo de investigaciones sobre educación secundaria, son todavía escasos los trabajos que se ocupan de la enseñanza con lxs estudiantes con discapacidad/es y porque, en su análisis de la articulación entre Educación Común y Educación Especial, las autoras ponen especial atención en las configuraciones relacionadas con la enseñanza.

Los procesos de articulación entre escuela secundaria común y escuela especial han sido poco estudiados desde el punto de vista tanto de sus facilitadores como de sus obstaculizadores desde una perspectiva inclusiva. El análisis que nos ofrecen Pitton y Demarco destaca la construcción de un trabajo colectivo entre Educación Común y Educación Especial, en el que se pone en evidencia la necesidad del encuentro entre saberes: "ninguno tiene por sí solo el saber completo y absoluto sobre cómo enseñar a los estudiantes con discapacidad". En la dinámica que reconstruyen, se recorta la forma en que la escuela interpreta la enseñanza: la Educación Especial no ocupa el lugar de apoyo especializado para la inclusión, sino que conforma con los docentes de la Educación Común la configuración que hace posible la participación de los estudiantes con discapacidad en las clases y en la vida cotidiana de la escuela. Así, uno de los hallazgos del estudio es el lugar que la enseñanza puede ocupar como eje estructurante en la construcción de la configuración de apoyo en la que participan la maestra integradora y los docentes de la escuela secundaria "común", lo que se documenta desde la voz de múltiples actores, incluso de lxs estudiantes. 


\section{Saberes clásicos y nuevos saberes}

La escuela secundaria tiene como uno de sus terrenos de crítica y conflicto la cuestión curricular. Heredero de la tradición del bachillerato y promotor de la cultura letrada (Dussel, 1997), el curriculum de la escuela secundaria, rigidizado por las condiciones de formación y contratación de lxs profesores, presenta importantes dificultades para incorporar nuevos saberes, los que, por su parte, suelen ser señalados como una incorporación necesaria para adecuar mejor la formación del nivel medio a los cambios en los modos de producción, circulación y clasificación de los conocimientos a lo largo del siglo XX y comienzos del siglo XXI. En este apartado, presentamos tres trabajos que se refieren a dos campos de "nuevos saberes" especialmente valorados en su posible aporte a la transformación de la educación secundaria: el primero se centra en los saberes propios de la cultura digital, en tanto los otros dos se refieren a la Educación Sexual Integral, de enseñanza obligatoria en las escuelas de todos los niveles en Argentina (Ley Nacional N ${ }^{\circ} 26.150$ ).

En "Diálogo de saberes entre la cultura escolar y la cultura digital en el Nivel Medio: oportunidades y desafíos", Rita de Pascuale y Sonia Sansot se proponen describir y comprender la complejidad de las prácticas de enseñanza en el Nivel Medio a partir del estudio de un instrumento de mediación específico, las Tecnologías de la Información y la Comunicación (TIC), en una investigación cuyo trabajo de campo se realizó en los últimos años de seis instituciones de Nivel Medio en las provincias de Neuquén y Río Negro.

Desde el marco teórico-metodológico de la tercera generación de la Teoría de la Actividad (véanse Engëstrom, 2001 y Daniels, 2001), se analizaron prácticas de enseñanza mediadas por TIC identificando Sistemas de Actividad entramados en una red: uno referido al sujeto de enseñanza, otro al sujeto de aprendizaje, y la emergencia de un tercero sobre competencias digitales. Como señalan las autoras, lo multimodal permea y se incorpora a la vida escolar, sin que se reconozca que medios y escuelas portan lógicas diferentes y ofrecen respuestas diferentes a las preguntas por el enseñar y el aprender. El desarrollo de prácticas letradas constituyó tradicionalmente uno de los objetivos centrales de la escolarización; las TIC amplían y complejizan el escenario con nuevos saberes cuyo dominio se ha vuelto crecientemente necesario.

Así como veremos, a propósito del trabajo de Delgado, Amud y Salas, una valoración positiva del aporte de la escuela con respecto a la formación para la ciudadanía democrática, también en el terreno de las TIC parece haber argumentos para sostener ciertas expectativas favorables. Señalan De Pascuale y Sansot la generalización del uso de las TIC, tanto por docentes como por estudiantes, a diferencia de lo que arrojaban estudios realizados en otros momentos, en los que las resistencias que expresaban los docentes a las incorporaciones de las TIC parecían mayores. Pero señalan también que en algunos casos se trata de una incorporación "fuera de planificación", "para hacer más agradable la clase, para captar la atención de sujetos de aprendizaje mediatizados", y no tanto para realizar un uso claramente instructivo. Dado que el trabajo se centra fundamentalmente en datos de encuestas y entrevistas, puede interrogarse si es posible que las respuestas de algunxs profesores reproduzcan discursos que circulan en la mayor parte de las escuelas del país a raíz de las acciones formativas que han acompañado los programas de acceso a conectividad y equipamiento.

Las interacciones y contradicciones entre los tres Sistemas de Actividad que identifican las autoras les permiten comprender el diálogo de saberes entre cultura digital y cultura escolar. La primera modifica los contextos de aprendizaje cotidianos e interpela a la segunda en sus modos de transmisión y construcción de saberes, formación y constitución de subjetividades. 
Con el trabajo de Catalina González del Cerro, "Transversalizar una perspectiva: voces, espacios y tiempos de la Educación Sexual Integral", nos adentramos en otro campo profundamente transformado en la cultura: el del reconocimiento de las identidades de género y la educación sexual integral (ESI) en las escuelas.

El propósito de artículo de González del Cerro es presentarnos una experiencia de trabajo sobre ESI en una escuela secundaria pública de la Ciudad de Buenos Aires, documentada en un estudio etnográfico, en el que se describen algunas de las acciones que llevaron adelante un grupo de estudiantes y docentes (autodenominado grupo ESI-Género) con el fin de sensibilizar a la comunidad en torno a las desigualdades de género; la autora reconstruye los modos en que el grupo tradujo a la vida cotidiana de su escuela la propuesta de transversalización de la perspectiva de género que promueve la normativa ESI. A partir de esta experiencia y de su análisis, la autora se propone reflexionar sobre los sentidos asignados a la transversalidad. La pregunta que guía su trabajo es sobre las condiciones de posibilidad para que la ESI sea transversal en la escuela. El riesgo de la transversalidad, nos dice González del Cerro, es la posible disolución o desdibujamiento de los aportes de la perspectiva de la ESI y de los feminismos en aquellas instituciones en las que ningún miembro de la comunidad "se hace cargo". La potencia, en cambio, radica en la posibilidad de transformar los mecanismos por los cuales se establece un reconocimiento del poder de las alianzas trans/intergeneracionales y trans/interdisciplinares. Un aporte original del trabajo es la reconstrucción que ofrece de los debates teóricos sobre la transversalidad a partir de hacer confluir las diversas definiciones que construyeron por separado la pedagogía, los estudios feministas y la normativa de la Educación Sexual Integral. Destaca también su conclusión acerca de la necesidad de fundamentar la dimensión institucional y no solo curricular de la enseñanza.

El trabajo de María Soledad Vázquez, "Educación Sexual Integral: reflexiones sobre experiencias institucionales en el nivel secundario", amplía los aportes del dossier para analizar el devenir escolar de la ESI desde la sanción de la Ley 26.150 en Argentina. En el artículo se ponen en diálogo dos experiencias contrastantes de Educación Sexual integral en escuelas de diferentes municipios de la provincia de Buenos Aires: la primera, en una institución secundaria de gestión privada de sectores socioeconómicos medios y medios-altos, laica pero con afinidades religiosas marcadas entre muchas de sus familias; la otra, desarrollada en un Bachillerato Popular del que participan jóvenes y adultos que atraviesan profundas vulnerabilidades materiales y simbólicas.

El trabajo pone de manifiesto las tensiones y conflictos que se suscitan en tiempos en que la normativa concibe a la escuela como un ámbito para reflexionar en torno de las sexualidades juveniles y las identidades sexuales no-normativas. El relato etnográfico y la descripción densa de escenas escolares en las dos instituciones permiten visualizar el rechazo de algunos estudiantes y sus familias al respeto por la diversidad y el ejercicio de los derechos, así como las consecuencias prácticas de los modos institucionales contrastantes de afrontar ese rechazo: una institución que evita la temática y otra que hace lugar al conflicto, habilitando un espacio específico de trabajo en torno de la ESI.

\section{Nuevamente, la secundaria como experiencia de formación}

En la primera parte de este dossier, que se publicó en el número 46 de la Revista, decíamos que, entre los muchos cuestionamientos de los que ha sido objeto la escuela Secundaria, el problema del sentido se recorta como un asunto principal. En aquella oportunidad, se presentaron tres trabajos que entraban de lleno en la cuestión de la experiencia formativa en la escuela secundaria, y que lo hacían identificando 
instituciones (escolares y no) que buscan hacer posibles experiencias que afectan al sujeto y su identidad. En este número se incorporan dos nuevos trabajos que permiten seguir elaborando reflexiones y conceptualizaciones acerca de las experiencias de formación que promueve la escuela secundaria. Uno de ellos estudia las representaciones de estudiantes próximos a egresar sobre la formación recibida en sus escuelas, seleccionadas bajo el supuesto de que funcionan como expresión de tantas otras; mientras que el segundo trabajo selecciona deliberadamente escuelas que promueven proyectos de producción de medios gráficos de comunicación, para poder documentar lo que produce en la experiencia de formación el ingreso de los saberes y problemáticas juveniles y el aprendizaje de otras formas de comunicación.

En "Sentidos y finalidades de la experiencia escolar de estudiantes de escuelas secundarias de Resistencia y Corrientes", Patricia Delgado, Cinthia Amud y Jorge Salas describen las visiones en torno a la escuela secundaria construidas por estudiantes que se encontraban terminando sus estudios en cuatro escuelas públicas de gestión estatal, dos de la ciudad de Corrientes y dos de Resistencia. Al estudiar, mediante cuestionarios semi-estructurados y grupos de discusión, cómo perciben y valoran la formación recibida en relación con las finalidades de la Secundaria, exploran la construcción del sentido que asume la experiencia escolar para ellos/as. El trabajo aporta cierta evidencia acerca de diferencias en los sentidos que se construyen entre quienes asisten a instituciones que son diferentes tanto en su modalidad como en la ubicación territorial, pero también de acuerdo al clima educativo y posición social de sus familias. Las diferencias las encuentran en las valoraciones que construyen los jóvenes con respecto a que la escuela los prepare para seguir estudiando o para insertarse en el mundo del trabajo. Para explicarlas, lxs autores consideran la influencia de las prácticas formativas que desarrollan los establecimientos, y también las representaciones y expectativas familiares con respecto a la continuidad de los estudios superiores, desde donde los y las estudiantes evalúan en forma positiva o negativa la formación recibida. Es interesante destacar que encuentran, en las cuatro escuelas de la muestra, que los niveles más altos de conformidad y consenso se registran con respecto al aprendizaje de valores, actitudes y normas para la convivencia social y el ejercicio de la ciudadanía democrática. Esta coincidencia en la valoración positiva que realizan estos/as estudiantes permite formular algunas expectativas acerca de la orientación que estaría tomando la formación en este nivel.

En el otro trabajo que compone este apartado, Verónica Plaza Schaefer analiza nuevas formas que puede tomar la experiencia escolar de lxs jóvenes en la escuela secundaria, al documentar los modos en que la condición juvenil asume un lugar protagónico en escuelas secundarias de la ciudad de Córdoba seleccionadas para su estudio porque generan proyectos que involucran la producción de medios gráficos de comunicación. El trabajo tiene un sugerente título: “Cuando 'ser joven' en la Escuela está permitido... Desplazamientos en la condición de estudiante a partir de, en escuelas secundarias de la ciudad de Córdoba". La autora señala una vacancia en la investigación sobre la escuela secundaria pues, en un contexto que describe signado por políticas educativas orientadas a incorporar lenguajes, tecnologías y medios de comunicación, advierte una significativa ausencia de registros sistemáticos que documenten las diferentes experiencias que se generan en los proyectos escolares. En la investigación que nos reporta, Plaza Schaefer encuentra que la realización de un medio de comunicación en la escuela permite abordar una significativa variedad de temáticas que trascienden y se articulan con las temáticas curriculares, y comunicarlas a partir de otras narrativas, formatos y estéticas. Estas dos características, que el trabajo analiza con aportes de datos de campo, favorecerían que los/as estudiantes sean "jóvenes" en la escuela, y propondrían un desplazamiento de la noción más tradicional de "alumno/a" hacia un modelo de estudiante más próximo a las prácticas que los/as jóvenes realizan en los otros ámbitos donde se desarrollan sus vidas. Encontramos, en el trabajo de Plaza 
Schaefer, algo similar a lo que traía el trabajo de Martínez, publicado en el número 46 de la Revista, a propósito de la formación en dos escuelas técnicas: son propuestas que aportan no solo saberes (sobre la comunicación, en las escuelas en las que se desarrolló el estudio de Schaefer Plaza; saberes técnicos, en las escuelas del trabajo de Martínez), sino también sentido a la escuela secundaria general y a la construcción subjetiva de quienes transitan por ella.

\section{Agradecimientos}

Tras más de un año de trabajo, la Revista del Instituto de Investigaciones en Ciencias de la Educación de la Facultad de Filosofía y Letras de la Universidad de Buenos Aires ha reunido y presenta veintiséis trabajos de investigación sobre Educación Secundaria que constituyen una importante referencia para la actualización del conocimiento en el campo. Quiero agradecer a las autoridades del Instituto y al Comité Editorial de la revista por su confianza en mí para coordinar este dossier, y a los colegas especialistas que actuaron como evaluadores de los numerosos artículos que hemos recibido. De todos los intercambios que hemos mantenido, así como de las evaluaciones que he tenido oportunidad de leer y que he tenido en cuenta para la elaboración de las dos presentaciones, me quedo con la certeza de un campo en expansión que aumenta su rigurosidad y las referencias compartidas. 


\section{Q Bibliografía}

» Arroyo, M.yPoliak, N.(2011). “Discusiones en torno a fragmentación, identidades y compromiso. Enseñar en las Escuelas de Reingreso". En Tiramonti, G. (Dir.), Variaciones sobre la forma escolar. Límites y posibilidades de la escuela media. Rosario: Homo Sapiens, Buenos Aires: FLACSO.

»Avelar, M. (2016). Entrevista con Stephen J. Ball: Su contribución al análisis de las políticas educativas (translated versión). Originalmente publicado como: Interview with S. J. Ball: Analyzing his contribution to education policy research. Archivos Analíticos de Políticas Educativas, 24, p. 24, doi: http://dx.doi. org/10.14507/epaa.24.2368

"Ball, S. J. y Bowe, R. (1992). Subject departments and the "implementation" of national curriculum policy: An overview of the issues. Journal of Curriculum Studies, 24 (2), pp. 97-115.

"Beech, J. y Meo, A. (2016). “Explorando el Uso de las Herramientas Teóricas de Stephen J. Ball en el Estudio de las Políticas Educativas en América Latina”. En Education Policy Analysis Archives/Archivos Analíticos de Políticas Educativas, vol. 24.

"Daniels, H. (2001). "Tendencias actuales en la teoría sociocultural y de la actividad” (Cap. 3). En Vigotsky y la pedagogía. Buenos Aires: Paidós.

»Di Pietro, S. y Abal Medina, M. de los D. (2013). "Cuando se busca la inclusión sin renunciar a la enseñanza: la experiencia de la escuela de reingreso". En Vázquez, S. (Coord.), Construir otra escuela secundaria. Aportes desde experiencias pedagógicas populares. Buenos Aires: La Crujía.

»Dussel, I. (1997). Curriculum, humanismo y democracia en la escuela media (18631920). Buenos Aires: Ediciones de la UBA/FLACSO.

"Engeström, Y. (2001). “Los estudios evolutivos del trabajo como punto de referencia de la teoría de la actividad: el caso de la práctica médica en la asistencia básica”. En Chaiklin, S. y Lave, J. (Comps.), Estudiar las Prácticas. Buenos Aires: Amorrortu.

» Gild, M. y Zyssholtz, F. (2017, 6 al 8 de septiembre). “La inclusión de jóvenes de sectores desfavorecidos en la escuela secundaria: desafíos al formato escolar tradicional", trabajo presentado en el VI Congreso Nacional e Internacional de Estudios Comparados en Educación: Inclusión, Calidad y Equidad en la Educación. Pasado, Presente y Futuro en perspectiva comparada. Buenos Aires. Recuperado de <www.saece.com.ar/doc/congreso6/trabo61.pdf> [último acceso 20 de julio de 2019].

» Krichesky, M.; Greco, M. y Saguier, V. (2015). “La experiencia en las Escuelas Medias de Reingreso de la Ciudad de Buenos Aires. Aportes de la investigación educativa para analizar los cambios de formato escolar y la perspectiva de sus actores". XI Jornadas de Sociología, Facultad de Ciencias Sociales, UBA, Buenos Aires.

" Maddonni, P. (2014). El estigma del fracaso escolar. Nuevos formatos para la inclusión y la democratización de la educación. Buenos Aires: Paidós.

» Terigi, F. (2008, junio). Los cambios en el formato de la escuela secundaria argentina: por qué son necesarios, por qué son tan difíciles. Propuesta educativa, 
17 (29), dossier “Reformas de la forma escolar”, pp. 63-71.

"Terigi, F. (2016). “Adolescentes y Educación Media/secundaria en América Latina: entre la expansión del modelo tradicional y las alternativas de baja escala". En Conferencias Magistrales del Congreso Nacional de Investigación Educativa, año 1, núm. 1, 2013- 2014. Iztapalapa: Consejo Mexicano de Investigación Educativa (COMIE), pp. 239-259.

»Terigi, F. (2020, en prensa). “La educación secundaria argentina 1983-2015”. En Puiggrós, A. (Dir.), Avatares en la historia reciente de la educación argentina (19832015). Buenos Aires: Galerna.

» Terigi, F.; Briscioli, B.; Scavino, C.; Morrone, A. y Toscano, A. G. (2014). La educación secundaria obligatoria en Argentina: entre la expansión del modelo tradicional y las alternativas de baja escala. Revista del Instituto de Investigaciones en Ciencias de la Educación, 33. Facultad de Filosofía y Letras, Universidad de Buenos Aires.

"Vanella, L.; Maldonado, M. (Eds.) (2013). Programa de Inclusión y Terminalidad de la Educación Secundaria para Jóvenes de 14 a 17 años (PIT) Córdoba (Argentina). Buenos Aires: UNICEF y Facultad de Filosofía y Humanidades, Universidad Nacional de Córdoba.

"Yapur, J. (2018). Nuevos formatos escolares: Igualdad de oportunidades, inclusión educativa y calidad: un estudio de caso del "Programa Inclusión / terminalidad del nivel secundario. (Tesis Doctoral). Doctorado en Educación, Facultad de Filosofía y Humanidades, Universidad Nacional de Córdoba. 
